\title{
An Incremental Discourse Parser Architecture
}

\author{
Dan Cristea \\ “Alexandru Ioan Cuza" University of Ia॰i, \\ Faculty of Computer Science \\ 16, Berthelot St., 6600 - Ia•i, Romania \\ dcristealinfoiasi.ro
}

\begin{abstract}
We present a discourse parsing architecture based on an incremental approach and aimed at building a rhetorical structure of a free text. Vein expressions computed on the developing structure help to restrict the domains of referential accessibility on which resolution of anaphora is performed. The parsing process is guided by cohesion and coherence constraints.
\end{abstract}

\section{Introduction}

Incremental discourse parsing is particularly challenging to systems aimed at doing automatic text processing because of its resemblance with the linear processing of texts done by humans.

Although humans process texts linearly, there are strong reasons to believe that they build structures which are not linear but hierarchical and that they use these structures to further resolve co-references and to build interpretations. Theories like Attentional State Theory [7], and Rhetorical Structure Theory [8] argued in favour of a hierarchical structure of discourse. There is debate whether the discourse structure must resonate with the resolution of anaphora (see for instance [13]). We believe there is tight relationship between discourse structure and references.

In [12] a system that integrates the expertise of several modules (syntactic, semantic, common sense inference, discourse planning, anaphora resolution, cuewords and temporal) in order to build incrementally the meaning of free texts was presented. The behaviour is based on the assumption that the performance of the system could be affected but is not vitally dependent on any of the contributing expert modules. The more one knows the more accurate the representation could be. If less is known, less is obtained, but the system can still survive.

In this paper we describe a configuration that integrates a part of speech tagger, a cue-expert module, a discourse parser and a reference resolution module. Section 2 presents the theoretical clues the approach is anchored in: Veins Theory and expectation-based incremental parsing, section 3 explains in detail the behaviour of the system on a particular fragment of English text and section 4 compares the proposed architecture with other work.

\section{Veins Theory}

Veins Theory [3] uses a representation of the discourse structure as a binary tree, close to the representation in Rhetorical Structure Theory (RST) [8]. Terminal nodes in the tree represent discourse units and non-terminal nodes represent continuous text spans and are labelled with rhetorical relations. Usually a label uniquely identifies a 
unit. A polarity is established among the children of a relation, which identifies at least one node, the nucleus, considered essential for the writer's purpose; non-nuclear nodes, which include spans of text that increase understanding but are not essential to the writer's purpose are called satellites. VT operates with two concepts: head and vein, which are expressions of terminal labels (discourse unit labels) attached to each node in the discourse structure. Head expressions (equivalent to Marcu's promotion sets [9]) are computed bottom-up:

- the head of a terminal node is its label;

- the head of a non-terminal node is the concatenation of the heads of its nuclear children.

Veins expressions are sub-sequences of the sequence of unit labels making up the discourse and are computed top-down:

- the vein expression of the root is its head expression;

- for each nuclear node whose parent node has vein $v$, the vein expression is:

- if the node has a left non-nuclear sibling with head $h$, then $\operatorname{seq}(\operatorname{mark}(h), v)$, where $\operatorname{mark}(x)$ is a function that takes a string of symbols $x$ and returns each symbol in $\mathrm{x}$ marked in some way (e.g. with parentheses) and $\operatorname{seq}(x, y)$ is a sequencing function that computes that permutation of $x$ concatenated with $y$ given by the left to right reading of the sequence of labels in $x$ and $y$ on the terminal frontier of the tree

- otherwise (if the node has no left sibling or its left sibling is nuclear), $v$;

- for each non-nuclear node of head $h$ whose parent node has vein $v$, the vein expression is:

- if the node is the left child of its parent, then $\operatorname{seq}(h, v)$

- otherwise, $\operatorname{seq}(h, \operatorname{simpl}(v))$, where $\operatorname{simpl}(x)$ is a function that eliminates all marked symbols from its argument, if they exist.

The definition for the vein expression of a node is intended to put in evidence a list of units of the discourse that make up a comprehensible context for the span of text covered by the node. In particular, the vein expression of the root node gives a summary of the whole discourse, while the vein expression of a terminal node - a unit of the discourse, reveals the minimum context around that unit that makes the unit understandable. The particular interest given to left satellites resides from empirical observations that they are accessible to their immediate to the right nuclei, but inaccessible to subsequent satellites.

The domain of referential accessibility (DRA) of a unit is defined as the string of unit labels appearing in its vein expression and prefixing that unit label itself.

\section{Ex. 1}

1. John sold his bicycle

2. although Bill would have wanted it.

3. He obtained a good price for it,

4. which Bill could have not afforded.

5. Therefore he decided to use the money for going in a trip.

The RST structure of Ex. 1, as put in evidence by the cue-words although, which, therefore, must be one of those evidenced in Fig. 1.

VT makes two important claims. The first regards discourse cohesion, the second discourse coherence. 

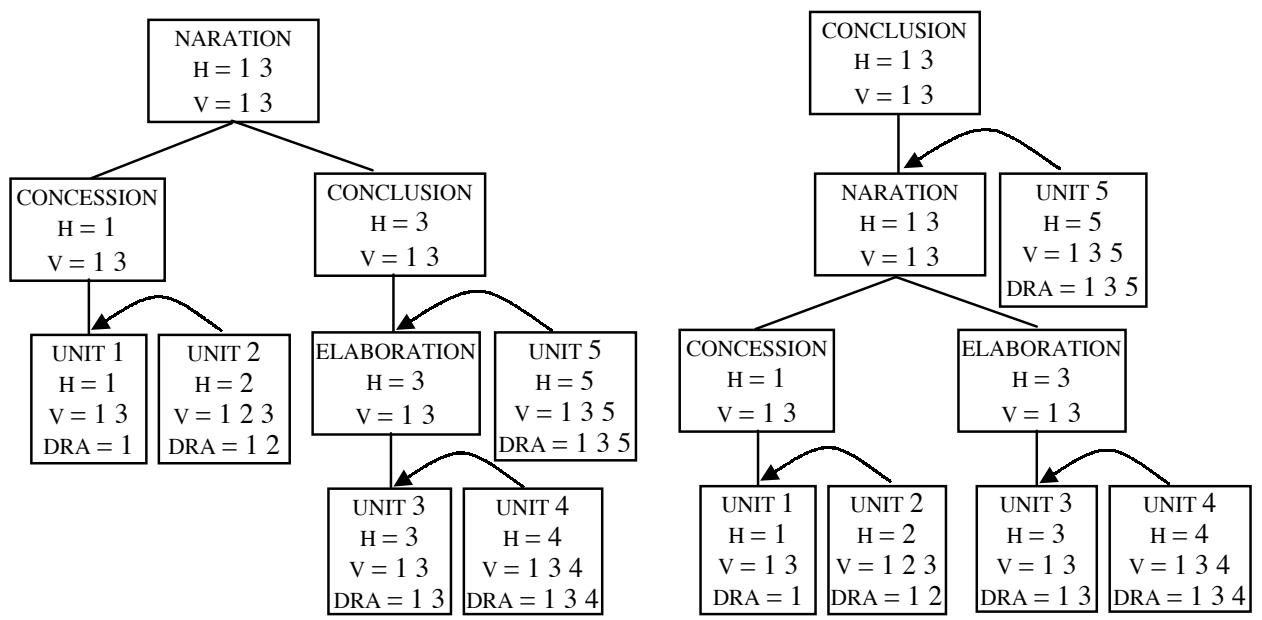

Fig. 1. Possible RST structures for Ex. 1

\subsection{VT and Discourse Cohesion}

Veins Theory claims that references from a given unit are possible mainly in its domain of referential accessibility. By restraining the domains the antecedents of anaphors are to be looked for, VT makes the existing anaphora resolution techniques [10] more effective and more efficient. The model we have studied for experimenting VT-driven anaphora resolution is based on a three tiered representation and a two steps process.

The first layer is that of the surface text. Referring expressions (REs) belong to this layer. The second layer is the one of the morpho-semantic restrictions. This layer is populated with feature structures that display sets of morphological and semantic feature-value pairs. To each RE on the textual layer correspond one or more representations on the morpho-semantic layer. We say that the morpho-semantic signs are projected there from the textual layer. The third layer is the situational layer, also populated with feature structures, but where all references are resolved. There is only one representation for each discourse entity on this layer. We say that the entities on the situational layer are evoked there from the restrictions layer (Fig. 2 displays the three layers for two co-referencing REs). Let's notice that the composition of the projects and evokes relations yields the realises relation of centering [6]. As such, the world of REs can be seen as a simplification of the overall dynamic model of language interpretation, where we retain only REs and representations emerged from them.

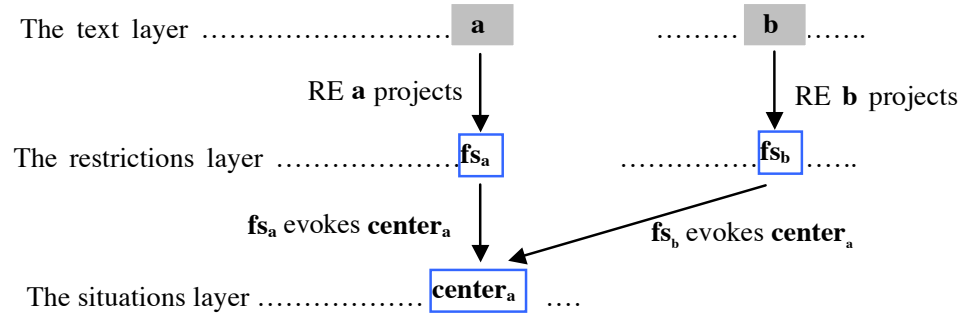

Fig. 2. The three-layer representation for two co-referencing REs 
Different theories of anaphora resolution use specific evoking mechanisms. For instance, the resolution of pronominal anaphors could impose the agreement in person, number, gender and a semantic feature (human or non-human). The evoking mechanism of noun phrase anaphors could be more complicated, as they could involve also synonymy and hipernymy relations as well as navigation processes in a WordNetlike conceptual hierarchy.

VT sees references in conjunction with discourse structure. The following types of references are put in evidence:

Direct references. If $A$ and $B$ are units in this textual order, $A$ belongs to the DRA of $B$ and $a \in A$ is linearly the most recent (to $B$ ) RE that realizes that same center as $b \in B$, we say that $b$ directly co-refers the center evoked by $a$ (see Fig. 3 ).

If $A$ and $B$ are units in this order, $A$ belongs in the DRA of $B$ and $a \in A$ is linearly the most recent RE (to $B$ ) that realises a role of $b \in B$, we say that $b$ functionally directly co-refers the center realised by $a$. To put in evidence the DRAs of units, in the following graphical representations the units and the veins they belong to are drawn above REs. Also, to simplify notation, only the first and the second layers are shown.

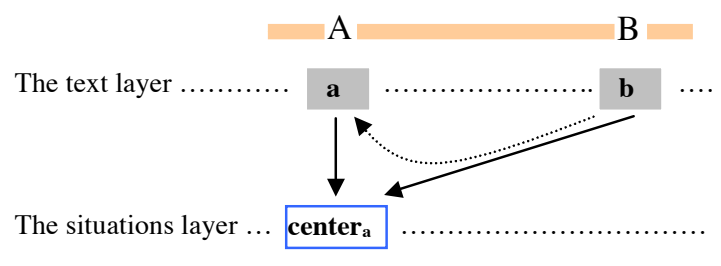

a. direct co-reference

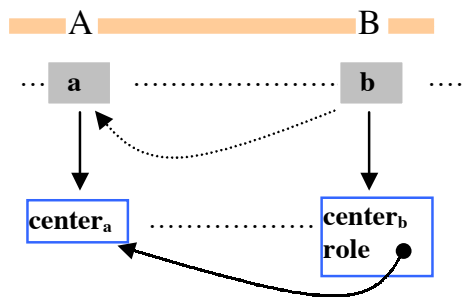

b. functional direct co-reference

Fig. 3. Direct co-reference: the linearly most recent RE that realises the same center of an RE is shown by a dashed arrow and the realisation relation by a full arrow

Indirect references. If $A, B$ and $C$ are units in this order, $b \in B$ is linearly the most recent (to $C$ ) RE that realises the same center as $c \in C, B$ is not on the DRA of $C, A$ is linearly the most recent (to $B$ ) unit that is both on the DRA of $B$ and of $C$, and it contains a RE $a \in A$ such that $b \in B$ realises the same center as $a$, we say that $c$ indirectly co-refers the center realised by $a$ (see ). A similar definition applies for indirect functional references.

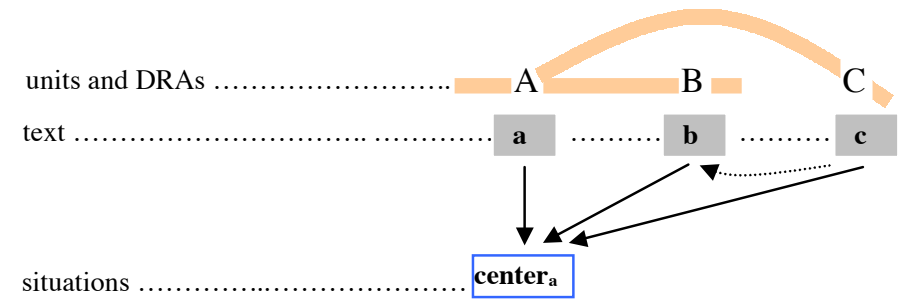

Fig. 4. Indirect co-reference

In other words, an indirect reference occurs when the DRA expression of the anaphor's unit intersects the one of the most recent antecedent, there is a unit on this common segment that realises the same center as the one referred by the anaphor and this unit is not linearly the most recent to the anaphor. In still another words, an 
indirect reference occurs when the chain of units of backward looking co-references intersects the DRA of anaphor's unit in a unit that is not the most recent to the anaphor's unit.

Anything that does not obey the direct or indirect references are inferential references. This happens when there is no intersection between the backward looking chain of co-references' units and the DRA of the anaphor's unit.

A particular category of inference references is something that we would call pragmatic references, or pseudo references. These are REs that practically do not refer back in the discourse although an entity identically realised was already introduced. If she says that she waked up this morning at the same time with the sun and, after a few sentences, she says that no cloud covers the sun, it is questionably that in this last sentence she refers back to her first mention of the celestial object. One can understand the last sentence without making the connection with the first one. We all know about sun and anytime we are said about it we know what the concept is because it is outside the text, it is the same to all of us, it is pragmatic knowledge. But if we make a story about the sun in which it is passing through different events, from its raising to its dawning, than here we have references. Although everybody knows what the sun is, one can understand the story only linking the textual entities mentioned in sequence to the same situational entity. Because they are told about in the same story, doing things or participating in connected events, it is important that one has the same mental representation about all the mentioned suns, and not only knowing them as having identical functionality.

The difference between cases as the ones mentioned could be reflected, although not necessarily, by considering the references in connection with the veins structure. It is most plausible that references in the first example could appear as inferential references, while references in the second example could be recovered as direct or indirect references.

Inferential references seem to minimize the importance of the domain of referential accessibility, as defined in VT, because references can now "escape" from the domain. Does anymore the domain of accessibility have any significance? Is it an artificial invention or it is defended by a natural characteristic of the manner humans process texts? We claim that there are two significantly distinct types of anaphora resolution processes: evocative (or associative) and post-evocative (or inferential).

The evocative resolution processes are due to direct and indirect references. They are based on associations, which are processes of pattern-matching on feature structures decorated with morpho-semantic attributes. They are performed between a feature structure projected by the anaphor RE and a center that exists already in the DRA of the unit the anaphor belongs to. As such, they are performed between layer two and layer three feature structures. These are fast processes, direct ones being faster and more frequent than indirect ones.

On the other hand, the post-evocative processes are inferential processes that are developed in memory, based on the knowledge accumulated by the preceding discourse, or based on the cultural knowledge the subject owns. We believe these inferences swings the semantic space in an order that is also dictated by discourse structure. Eventually, the target entity can be found based on a pattern-matching process between the projected structure of the anaphor and the center of the antecedent. They are slow (compel to more inference load), necessitate more powerful referencing means (like proper nouns) and are less frequent processes.

Fig. 5 shows different types of anaphoric relations for the discourse of Ex. 1. 


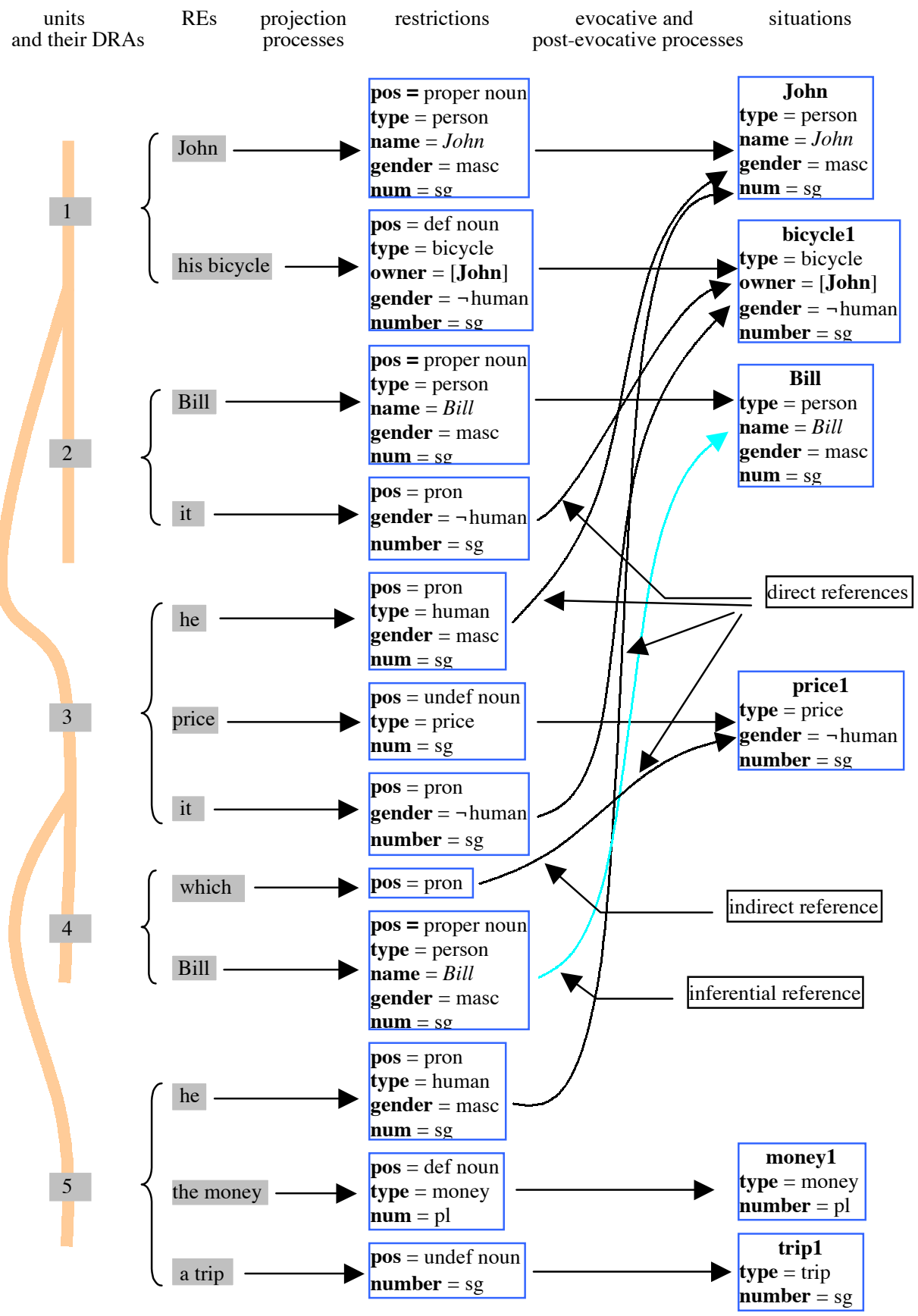

Fig. 5. Anaphoric relations in connection with discourse structure on Ex. 1 


\title{
2.2 VT and Discourse Coherence
}

The second claim of VT extends the classical Centering Theory (CT) [6] at global level. As defined, centering is a theory of local coherence. CT defines a set of transition types for discourse [6], [1]: CONTINUATION which is easier to process than RETAINING, which is easier than SMOOTH SHIFTING, which is easier than ABRUPT SHIFTING. In [3] a smoothness index was introduced to compare different discourse structures and interpretations. The smoothness index for a transition is based on the CT natural rating of transitions: CONTINUATION $=4$, RETAINING $=3$, SMOOTH SHIFTING $=2$, ABRUPT SHIFTING $=1$, no backward-looking center $(\mathrm{Cb})=0$. Then the scores for each transition in the entire segment are summed up, and the result is divided by the number of transitions in the segment. An index of the overall coherence of the segment, called global smoothness score, is such obtained. VT claims that the global smoothness score of a discourse when computed following the neighbouring metric given by vein expressions, following VT, is at least as high as the score computed following the adjacency metric recommended by CT, when extended to the whole discourse. By this, VT claims that long-distance transitions computed over returnpops, using vein expressions, are systematically smoother than accidental transitions at segment boundaries. We use the global smoothness score as criteria of acceptability of a discourse structure.

Table 1 and Table 2 exemplify transitions and global scores in CT and, respectively, VT for Ex. 1. A comparison between the two tables clearly show the better global score obtained in the hierarchical case. The three sequences of units form coherent argumentation lines:

\author{
1. John sold his bicycle \\ 2. although Bill would have wanted it. \\ 1. John sold his bicycle \\ 3. He obtained a good price for it, \\ 4. which Bill could have not afforded. \\ 1. John sold his bicycle \\ 3. He obtained a good price for it, \\ 5. Therefore he decided to use the money for going in a trip.
}

and, on each of them, the discourse is smoother then in the original overall discourse.

Table 1. Transitions and scores on a linear adjacency metric $(\mathbf{J}=[\mathbf{J o h n}], \mathbf{b}=[\mathbf{J o h n}$ 's bicycle $]$, $\mathbf{B}=[$ Bill $], \mathbf{p}=[$ price $], \mathbf{m}=[$ the money $], \mathbf{t}=[\mathbf{a}$ trip $]$ )

\begin{tabular}{|c|c|c|c|c|c|}
\cline { 2 - 6 } \multicolumn{1}{c|}{} & 1 & 2 & 3 & 4 & 5 \\
\hline CF & $\mathbf{J}, \mathbf{b}$ & $\mathbf{B}, \mathbf{b}$ & $\mathbf{J}, \mathbf{p}, \mathbf{b}$ & $\mathbf{p}, \mathbf{B}$ & $\mathbf{J}, \mathbf{m}, \mathbf{t}$ \\
\hline CB & $\mathbf{J}$ & $\mathbf{b}$ & $\mathbf{b}$ & $\mathbf{p}$ & - \\
\hline Trans & & $\mathrm{ASH}$ & $\mathrm{RET}$ & $\mathrm{SSH}$ & no Cb \\
\hline Score & & 1 & 3 & 2 & 0 \\
\hline Global & & & & & $6 / 4=1.5$ \\
\hline
\end{tabular}


Table 2. Transitions and scores on a hierarchical adjacency metric (same notations as in Table 1). Only one transition is computed for each unit (excepting the first)

\begin{tabular}{|c|c|c|}
\cline { 2 - 3 } \multicolumn{1}{c|}{} & 1 & 2 \\
\hline CF & $\mathbf{J}, \mathbf{b}$ & $\mathbf{B}, \mathbf{b}$ \\
\hline CB & $\mathbf{J}$ & $\mathbf{b}$ \\
\hline Trans & & ASH \\
\hline Score & & 1 \\
\hline Global & & \\
\hline
\end{tabular}

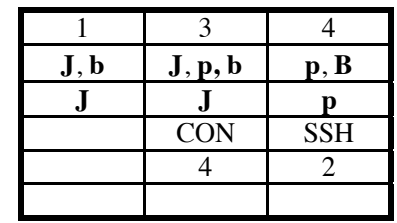

\begin{tabular}{|c|c|c|}
\hline 1 & 3 & 5 \\
\hline $\mathbf{J}, \mathbf{b}$ & $\mathbf{J}, \mathbf{p}$ & $\mathbf{J}, \mathbf{m}, \mathbf{t}$ \\
\hline $\mathbf{J}$ & $\mathbf{J}$ & $\mathbf{J}$ \\
\hline & $\mathrm{CON}$ & $\mathrm{CON}$ \\
\hline & & 4 \\
\hline & & $11 / 4=2.75$ \\
\hline
\end{tabular}

\subsection{Incremental Discourse Parsing}

Expectation-driven incremental discourse parsing [4] applies to the processing of discourse an idea of incremental parsing developed initially for syntactic analysis within the LTAG framework [11]. Two operations on trees, adjunction and substitution, add at each step a small piece of structure to the current developed tree (CDT). Adjoining adds an auxiliary tree with at least one material (discourse unit) node. Substitution unifies the root of a substitution structure with an empty node (denoting an expectation) of a CDT. These operations can be performed only on the generalised right frontier of the CDT, equal to the right frontier if CDT is expectation nodes free, or the right frontier of the subtree rooted by the left sibling of the innermost expectation node, otherwise.

\section{A Mixed Approach in Discourse Parsing}

Using VT as a guiding theory in an incremental discourse parsing approach is based on the assumption that the tree resulted after the parsing process, among the family of all possible RST-like discourse trees composed with rhetorical relations induced by cue-words of the text, is the one which manifests the more natural overall references over the discourse structure and the smoothest overall CT transitions on veins. The computation of centering transitions is based heavily on the resolution of anaphoric expressions which are looked for within the domains of accessibility given by the vein expressions in the developing tree.

In what follows we investigate the behaviour of a system with a more restricted architecture than the one presented in [12]. From the set of optional knowledge sources we retain only four: a part-of-speech tagger (PosTag) - which provides morphological markers for each word, a cue-words expert (CueExp) - which splits the text in discourse units ${ }^{1}$ and provides hints with respect to identification of rhetorical relations and their place of insertion based on a collection of cue-words heuristics, a reference resolution expert (RefExp) - aiming to solve inter-unit pronominal and functional anaphora, and an incremental discourse parser (DiscPar) - which builds the associated discourse tree(s).

On the text in Ex. 1 the incremental parser performs as shown in Fig. $6^{2}$. The first step builds the trivial tree made of only one unit. As this unit initialises the discourse, RefExp will introduce two semantic entities (centers) corresponding to John and his bicycle, as in Fig. 5.

\footnotetext{
${ }^{1}$ Minimally a clause, maximally a dot-to-dot compound sentence.

${ }^{2}$ To make the text shorter, some details are skipped.
} 
Based on the cue-word although, CueExp proposes for the auxiliary tree of the second unit a CONCESSION relation and the discourse parser adjoins it in the unique node of the right frontier - unit 1 (in Fig. 6 nodes of the right frontier are numbered in sequence bottom-up - 0 being the lowest right one). RefExp will find that the projected FS of Bill doesn't match any of the centers [John] and [bicycle1], both placed on the vein of unit 2, the only existing FS on the semantic layer at the moment, and this will result in introducing a new entity, [Bill]. As for it, it will match [bicycle1].

Step 1

Step 2

$\mathrm{CDT}_{1}: 1$

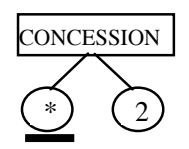

adjoining in node 0

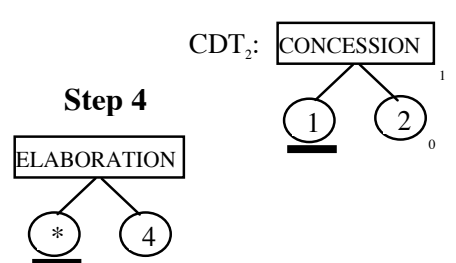

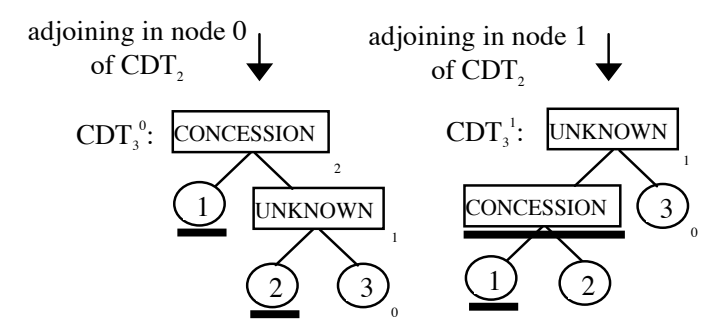

adjoining in node 0 of $\mathrm{CDT}_{3}{ }^{1}$

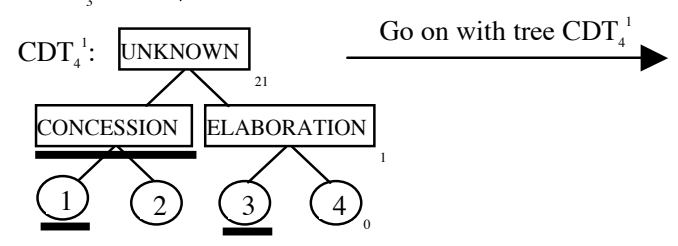

ing in node 0

of $\mathrm{CDT}_{2}$

of $\mathrm{CDT}_{2}$

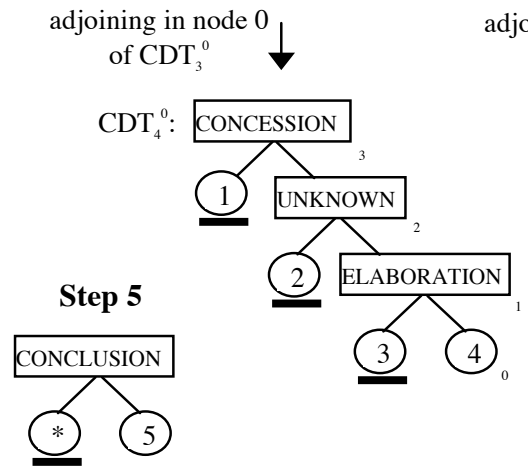

Step 3
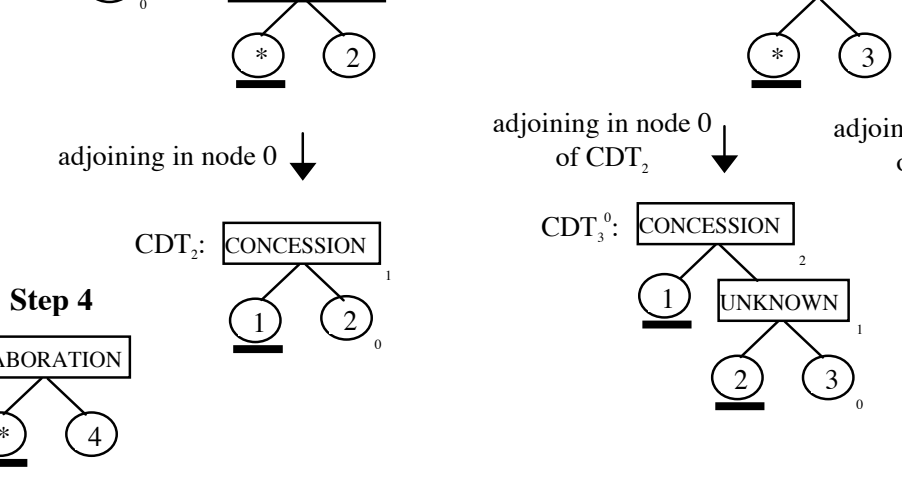

ining in node 0

$\mathrm{CDT}_{4}^{0}$ : CONCESSION

adjoining in node 0 of $\mathrm{CDT}_{4}{ }^{1}$

adjoining in node 1 of $\mathrm{CDT}_{4}{ }^{1}$

$$
\mathrm{CDT}_{5}{ }^{0} \text { : UNKNOWN }
$$

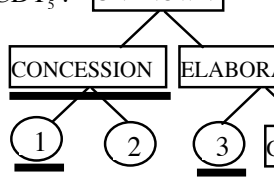

$$
\mathrm{CDT}_{5}{ }^{1} \text { : UNKNOWN }
$$

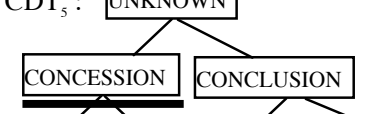

CONCLUSION

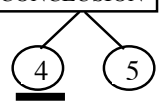

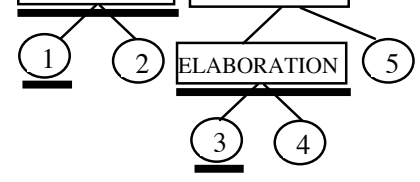

adjoining in node 2 of $\mathrm{CDT}_{4}{ }^{1}$

$\mathrm{CDT}_{5}^{2}$ : CONCLUSION

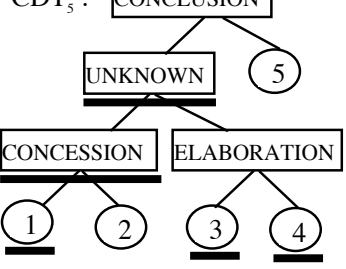

Fig. 6. Incremental tree building for the discourse in Ex. 1. 
There is no cue word in unit 3 that could hint on the type of relation, so CueExp proposes an UNKNOWN relation as root of the auxiliary tree. At step 3 there are two places (marked 0 and 1) on the right frontier of the $\mathrm{CDT}_{2}$ where this auxiliary tree could be adjoined by DiscPar. Adjoining at node 0 would raise $\mathrm{CDT}_{3}{ }^{0}$ such that $\operatorname{vein}(3)=123$, while adjoining at node 1 yields $\mathrm{CDT}_{3}{ }^{1}$ where vein $(3)=13$. RefExp is trying to resolve the anaphors using the DRAs given by VT on the already developed tree. As the domain of unit 3 on $\mathrm{CDT}_{3}{ }^{1}$ is 13 , the REs he, price and it of this unit will be matched against semantic entities introduced by REs of units 3 and 1 (in this order) and will, therefore, be found to be [John], [price1] (no match, newly introduced) and [bicycle1], respectively. Note that the pronoun he will not be erroneously linked to the entity [Bill] with whom it resonate and which is linearly more recent. In the variant of $\mathrm{CDT}_{3}{ }^{0}$ the domain of unit 3 is 123 and both [John] and [Bill] are candidates for being referents of he, while it could refer the same [bicycle1] as before. When he is chosen to evoke [John] the centering transition from 2 to 3 is ABRUPT SHIFTING while if he would be [Bill], the transition is CONTINUING. Therefore on $\mathrm{CDT}_{3}^{0}$ he in 3 is more plausible to be [Bill] than [John] (obviously an erroneous choice), while in $\mathrm{CDT}_{3}{ }^{1}$ he is unequivocally [John]. At this moment the parser has two structures that will continue to be developed in parallel until one is significantly better scored than the other.

At step 4 CueExp founds the cue word which to be an indication that this unit is an ELABORATION of the preceding one. Therefore on $\mathrm{CDT}_{3}^{0}$ only one place of the three on the right frontier is found of interest, while on $\mathrm{CDT}_{3}{ }^{1}-$ only one of the two. The resulting trees are $\mathrm{CDT}_{4}{ }^{0}$ that gives vein(4) =1234 and $\mathrm{CDT}_{4}{ }^{1}$ with vein(4) $=134$. The variant when he in unit 3 is bound to [Bill] would result in the case of $\mathrm{CDT}_{4}^{0}$ to the extremely low probable reference (see [5]) from the proper noun Bill in unit 4 to the entity [Bill] realised by a pronoun in unit 3 . This makes that at this moment, of the two possible variants of $\mathrm{CDT}_{4}{ }^{0}$, to be retained the one that indicated he in 3 being bound to [John] and Bill in 4 referring back in unit 2 to [Bill]. On the tree $\mathrm{CDT}_{4}{ }^{1}$ the same links are found, although now unit 2, not being on the vein of unit 4 , the reference Bill from 4 to 2 is of an inference nature, still plausible (being realised through a proper noun). The overall smoothness scores of the trees $\mathrm{CDT}_{4}{ }^{0}$ and $\mathrm{CDT}_{4}{ }^{1}$, are compared (6-on the sequence 1234 as in Table 1, versus 7 - on the sequences 1 2 and 134 as in Table 2) and the best is retained: $\mathrm{CDT}_{4}{ }^{1}$.

The last step exploits the cue-word therefore of unit 5 . There are three places on the right frontier of the $\mathrm{CDT}_{4}{ }^{1}$ where the adjoining of the auxiliary tree headed by a CONCLUSION relation could be operated (marked 0,1 and 2 on Fig. 6). As CueExp gives no indication as to where to place the aux tree, three final trees will be obtained. A possible rating is done in correlation with the resolution of the pronoun he and the definite noun the money. On $\mathrm{CDT}_{5}{ }^{0} \operatorname{vein}(5)=1345$, on $\mathrm{CDT}_{5}{ }^{1} \operatorname{vein}(5)=135$ and on $\mathrm{CDT}_{5}^{2}$ vein $(5)=15$.

$\mathrm{CDT}_{5}{ }^{0}$ where he in 5 is [Bill] is a rather strange interpretation where the money are those Bill intended to give for buying John's bike, and because they were not enough he decided to use them for going in a trip. $\mathrm{CDT}_{5}{ }_{5}^{1}$ gives an interpretation where the decision of John to use the money for going in a trip came as a result of John obtaining a good price for his bike, and is inferable that the money are the money John obtained for the bike. $\mathrm{CDT}_{5}^{2}$ evokes the interpretation in which the decision to go in a trip is only a result of John selling the bike. The money are again those obtained for the bike. The CT transitions rates for the three interpretations are given in the following tables: 
Table 3. Transitions corresponding to $\mathrm{CDT}_{5}{ }^{0}$ and he in $5=[\mathrm{Bill}]$

\begin{tabular}{|c|c|c|}
\cline { 2 - 3 } \multicolumn{1}{c|}{} & 1 & 2 \\
\hline CF & $\mathbf{J}, \mathbf{b}$ & $\mathbf{B}, \mathbf{b}$ \\
\hline CB & $\mathbf{J}$ & $\mathbf{b}$ \\
\hline Trans & & ASH \\
\hline Score & & 1 \\
\hline Global & & \\
\hline
\end{tabular}

\begin{tabular}{|c|c|c|c|}
\hline 1 & 3 & 4 & 5 \\
\hline $\mathbf{J}, \mathbf{b}$ & $\mathbf{J}, \mathbf{p}, \mathbf{b}$ & $\mathbf{p}, \mathbf{B}$ & $\mathbf{B}, \mathbf{m}, \mathbf{t}$ \\
\hline $\mathbf{J}$ & $\mathbf{J}$ & $\mathbf{p}$ & $\mathbf{B}$ \\
\hline & $\mathrm{CON}$ & $\mathrm{SSH}$ & $\mathrm{SSH}$ \\
\hline & 4 & 2 & 2 \\
\hline & & & $9 / 4=2.25$ \\
\hline
\end{tabular}

Table 4. Transitions corresponding to $\mathrm{CDT}_{5}{ }_{5}$ and he in $5=[\mathbf{J o h n}]$

\begin{tabular}{|c|c|c|}
\cline { 2 - 3 } \multicolumn{1}{c|}{} & 1 & 2 \\
\hline CF & $\mathbf{J}, \mathbf{b}$ & $\mathbf{B}, \mathbf{b}$ \\
\hline CB & $\mathbf{J}$ & $\mathbf{b}$ \\
\hline Trans & & ASH \\
\hline Score & & 1 \\
\hline Global & & \\
\hline
\end{tabular}

\begin{tabular}{|c|c|c|c|}
\hline 1 & 3 & 4 & 5 \\
\hline $\mathbf{J}, \mathbf{b}$ & $\mathbf{J}, \mathbf{p}, \mathbf{b}$ & $\mathbf{p}, \mathbf{B}$ & $\mathbf{J}, \mathbf{m}, \mathbf{t}$ \\
\hline $\mathbf{J}$ & $\mathbf{J}$ & $\mathbf{p}$ & - \\
\hline & CON & SSH & no Cb \\
\hline & 4 & 2 & 0 \\
\hline & & & $7 / 4=1.75$ \\
\hline
\end{tabular}

Table 5. Transitions corresponding to $\mathrm{CDT}_{5}{ }^{1}$ and he in $5=[\mathbf{J o h n}]$

\begin{tabular}{|c|c|c|}
\cline { 2 - 3 } \multicolumn{1}{c|}{} & 1 & 2 \\
\hline CF & $\mathbf{J}, \mathbf{b}$ & $\mathbf{B}, \mathbf{b}$ \\
\hline CB & $\mathbf{J}$ & $\mathbf{b}$ \\
\hline Trans & & ASH \\
\hline Score & & 1 \\
\hline Global & & \\
\hline
\end{tabular}

\begin{tabular}{|c|c|c|}
\hline 1 & 3 & 4 \\
\hline $\mathbf{J}, \mathbf{b}$ & $\mathbf{J}, \mathbf{p}, \mathbf{b}$ & $\mathbf{p}, \mathbf{B}$ \\
\hline $\mathbf{J}$ & $\mathbf{J}$ & $\mathbf{p}$ \\
\hline & $\mathrm{CON}$ & $\mathrm{SSH}$ \\
\hline & 4 & 2 \\
\hline & \multicolumn{2}{|l}{} \\
\hline
\end{tabular}

\begin{tabular}{|c|c|c|}
\hline 1 & 3 & 5 \\
\hline $\mathbf{J}, \mathbf{b}$ & $\mathbf{J}, \mathbf{p}, \mathbf{b}$ & $\mathbf{J}, \mathbf{m}, \mathbf{t}$ \\
\hline $\mathbf{J}$ & $\mathbf{J}$ & $\mathbf{J}$ \\
\hline & $\mathrm{CON}$ & $\mathrm{CON}$ \\
\hline & & 4 \\
\hline & & $11 / 4=2.75$ \\
\hline
\end{tabular}

Table 6. Transitions corresponding to $\mathrm{CDT}_{5}^{2}$ and he in $5=[\mathbf{J o h n}]$

\begin{tabular}{|c|c|c|}
\cline { 2 - 3 } \multicolumn{1}{c|}{} & 1 & 2 \\
\hline CF & $\mathbf{J}, \mathbf{b}$ & $\mathbf{B}, \mathbf{b}$ \\
\hline CB & $\mathbf{J}$ & $\mathbf{b}$ \\
\hline Trans & & ASH \\
\hline Score & & 1 \\
\hline Total & & \\
\hline
\end{tabular}

\begin{tabular}{|c|c|c|}
\hline 1 & 3 & 4 \\
\hline $\mathbf{J}, \mathbf{b}$ & $\mathbf{J}, \mathbf{p}, \mathbf{b}$ & $\mathbf{p}, \mathbf{B}$ \\
\hline $\mathbf{J}$ & $\mathbf{J}$ & $\mathbf{P}$ \\
\hline & $\mathrm{CON}$ & $\mathrm{SSH}$ \\
\hline & 4 & 2 \\
\hline & \multicolumn{2}{|l}{} \\
\hline
\end{tabular}

\begin{tabular}{|c|c|}
\hline 1 & 5 \\
\hline $\mathbf{J}, \mathbf{b}$ & $\mathbf{J}, \mathbf{m}, \mathbf{t}$ \\
\hline $\mathbf{J}$ & $\mathbf{J}$ \\
\hline & $\mathrm{CON}$ \\
\hline & 4 \\
\hline & $11 / 5=2.75$ \\
\hline
\end{tabular}

The scores given in these tables are used to rate the trees obtained. The final trees of the analysis are thus $\mathrm{CDT}_{5}{ }^{1}$ and $\mathrm{CDT}_{5}{ }^{2}$, very close to the ones in Fig. 1.

\section{Conclusions}

In this paper we revise Veins Theory - a theory of discourse cohesion and coherence and show how it can be used in an architecture aimed at building discourse structure simultaneously with resolving anaphorae. The discourse units are revealed from raw text by a collection of heuristics that rely on cue words. Units are incrementally attached to the developing tree on the right frontier. By computing the vein expression and the domain of referential accessibility of the current unit, the search for antecedents of anaphorae belonging to this unit is restricted. The resolution of anaphorae permits determination of centering transitions using the hierarchical adjacency metric given by veins. Transitions are then used to restrain the exponential 
explosion of the obtained trees by sorting the trees in the descending order of a global smoothness score and retaining only the most relevant ones for further processing.

Further work is necessary in order to establish the generosity of this approach compared to others (as the one in [9], for instance, that is very well suited for learning parser's moves from a corpus). Automatic learning of heuristics for splitting the discourse in units from an annotated corpus is equally possible in our approach. It remains to establish the feasibility of applying learning techniques for acquiring heuristics that would indicate shapes of auxiliary trees and the places of their adjunction on the developing tree.

\section{References}

1. Brennan, S.E., Walker Friedman, M., Pollard, C.J.: A centering approach to pronouns. Proc. of the $25^{\text {th }}$ Annual Meeting of ACL, Stanford (1987)

2. Cristea, D., Ide, N., Marcu, D., Tablan, M.V.: Discourse structure and CoReference: An Empirical Study. In: Proceedings of the Workshop Relations Between Discourse Structure and Reference, ACL Maryland (1999)

3. Cristea, D., Ide, N., Romary, L.: Veins Theory - A Model of Global Discourse Cohesion and Coherence. In: Proc. of Coling/ACL'98, Montreal (1998)

4. Cristea, D. Webber, B.L.: Incremental expectations in discourse parsing. In: Proc. of ACL/EACL, Madrid (1997)

5. Gordon P.C., Hendrick R.: The representation and processing of coreference in discourse. In: Cognitive Science, 22 (1998)

6. Grosz, B.J.; Joshi, A., Weinstein, S.: Centering - a framework for modelling the local coherence of discourse. In: Computation Linguistics, 12(2), June (1995)

7. Grosz, B., Sidner, C.: Attention, intention and the structure of discourse. In: Computational Linguistics, 12 (1986)

8. Mann, W.C., Thompson S.A.: Rhetorical structure theory: A theory of text organization. In: Text 8:3 (1988)

9. Marcu, D.: A Decision-Based Approach to Rhetorical Parsing. In: Proceedings of the ACL'99 (1999)

10. Mitkov, R.: Robust Pronoun Resolution with Limited Knowledge. In: Proceedings of COLING-ACL'98, Montreal (1998)

11. Shabes, Y.: Mathematical and Computational Aspects of Lexicalized Grammars, Technical Report MS-CIS-90-48, LINC LAB 179 (1990)

12. Tablan, M.V., Barbu, C., Popescu, H., Hamza, R.O., Ciobanu, C., Ni॰•, I.C., Bocønial•, C.D., Georgescul, M., Cristea D.: Co-opertion and Detachement in Discourse Understanding. In: Proc. of the Workshop on Lexical Semantics and Discourse, ESSLLI'98, Saarbruecken (1988)

13. Walker M.A.: Centering, Anaphora Resolution and Discourse Structure. In Walker, M.A.; Joshi, A.K.; Prince, E.F. (eds.): Centering in Discourse, Oxford University Press (1997) 